\title{
Protective effect of controlled release of cytokine response modifier A from chitosan microspheres on rat chondrocytes from interleukin-1 $\beta$ induced inflammation and apoptosis
}

\author{
PANG-HU ZHOU ${ }^{1 *}$, LEI SHI $^{2 *}$ and BO QIU ${ }^{1}$ \\ Departments of ${ }^{1}$ Orthopedics and ${ }^{2}$ Oncology, Renmin Hospital of Wuhan University, Wuhan, Hubei 430060, P.R. China
}

Received August 21, 2016; Accepted May 11, 2017

DOI: $10.3892 /$ etm.2017.4884

\begin{abstract}
The aim of the present study was to investigate the protective effect of cytokine response modifier A ( CrmA) released from chitosan (CS) microspheres in a controlled manner on interleukin (IL)-1 $\beta$-induced inflammation and apoptosis in chondrocytes. The $\mathrm{CrmA}$ release kinetics were characterized by an initial burst release, which was reduced to a linear release over 8 days. Furthermore, chondrocytes were isolated from 1-week-old Sprague Dawley rats. The cell culture was established by stimulation with $10 \mathrm{ng} / \mathrm{ml} \mathrm{IL-1} \beta$ and subsequent incubation with CS-CrmA microspheres. Following stimulation with IL-1 $\beta$, the viability of chondrocytes was decreased. However, the cell viability was attenuated by CS-CrmA microspheres as revealed by a cell counting kit-8 assay. CS-CrmA microspheres significantly inhibited IL-1 $\beta$-induced inflammation in chondrocytes by attenuating increases in the gene expression levels of inducible nitric oxide synthase and cyclooxygenase- 2 , as well as the concentrations of nitric oxide and prostaglandin E2. CS-CrmA microspheres significantly decreased the number of apoptotic chondrocytes induced by IL-1 $\beta$ as indicated by a terminal deoxyribonucleotide transferase deoxyuridine triphosphate nick-end labeling assay. In addition, CS-CrmA microspheres blocked IL-1 $\beta$-induced chondrocyte apoptosis by increasing B-cell lymphoma 2 (Bcl-2) and decreasing Bcl-2-associated $\mathrm{X}$ protein, caspase- 3 and poly adenosine diphosphate-ribose polymerase expression at the mRNA and protein levels, as indicated by reverse-transcription quantitative polymerase chain reaction and western blot analysis, respectively. The results of the present study revealed that CS-CrmA microspheres, as a controlled release system of CrmA, may protect
\end{abstract}

Correspondence to: Professor Bo Qiu, Department of Orthopedics, Renmin Hospital of Wuhan University, 238 Liberation Road, Wuhan, Hubei 430060, P.R. China

E-mail: qiuboprofessor@163.com

*Contributed equally

Key words: controlled-release, cytokine response modifier A, chitosan, interleukin $1 \beta$, chondrocyte rat chondrocytes from IL-1 $\beta$-induced inflammation and apoptosis via regulating inflammatory and apoptosis-associated genes.

\section{Introduction}

Osteoarthritis (OA) is an age-associated joint disease that is characterized by the degeneration of articular chondrocytes, leading to osteophyte formation and subchondral bone sclerosis and producing pain and loss of function (1). Chondrocytes are only one cell type, which are responsible for the maintenance of the extracellular matrix (2). Interleukin (IL)-1 $\beta$ has been reported to have important roles in OA and stimulation with IL-1 $\beta$ is able to upregulate the expression of nitric oxide synthase (iNOS) and cyclooxygenase-2 (COX-2) in chondrocytes, which promotes the production of nitric oxide (NO) and prostaglandin E2 $\left(\mathrm{PGE}_{2}\right)(3,4)$. Apoptosis is known to be important in the pathophysiological process of $\mathrm{OA}$ and IL-1 $\beta$ has been reported to accelerate aging and increase the apoptotic index of chondrocytes $(5,6)$. Therefore, inhibition of the IL-1 $\beta$ pathway that activates inflammation and apoptosis is of great importance for cell apoptosis inhibition in chondrocytes during OA pathogenesis. One of the major endogenous inhibitors of the IL-1 $\beta$ pathway is the cytokine response modifier A (CrmA), which is able to bond with the IL-1 $\beta$ converting enzyme (ICE) as a pseudosubstrate (7). This serpin can prevent the proteolytic activation of IL-1 $\beta$ and subsequently block the cleavage of pro-IL-1 $\beta$ by ICE, thereby suppressing an IL-1 $\beta$ response to infection and decreasing the secretion of IL-1 $\beta(8,9)$.

Chitosan (CS) is obtained by deacetylation of its parent polymer chitin, a polysaccharide that is widely distributed in nature (10). It has been reported to be non-toxic and bioabsorbable, and is known to promote wound healing and contribute to the maintenance of the chondrogenic phenotype, particularly in its morphology (11-13). Additionally, CS was widely used to elaborate different nanocarriers attributed to the capacity of the polymer to interact with negatively charged cell surfaces $(14,15)$. It has been widely used in drug, DNA delivery and tissue engineering due to its non-toxicity, biocompatibility and biodegradability (16-18). Furthermore, it promotes attachment, proliferation and viability of mesenchymal stem cells, and due to these features, CS and its derivatives are considered as promising biomaterials (19). 
The present study attempted to use CS to develop CrmA-carrying microspheres and to investigate their effect against IL-1 $\beta$-induced inflammation and apoptosis in rat chondrocytes by establishing an in vitro model of OA. The results indicated that CS-CrmA microspheres efficiently released CrmA to attenuate chondrocyte inflammation and apoptosis and may be suitable for the treatment of OA.

\section{Materials and methods}

Reagents. CS (molecular weight, $150 \mathrm{kDa}$; deacetylation, 98\%) and sodium tripolyphosphate (STPP) were provided by Sigma-Aldrich. (Merck KGaA, Darmstadt, Germany). Recombinant rat IL-1 $\beta$ and CrmA were purchased from PeproTech, Inc. (Rocky Hill, NJ, USA). Trypsinase, collagenase II, Dulbecco's modified Eagle's medium (DMEM)/F12, fetal bovine serum (FBS), 6-diamidino-2-phenylindole dihydrochloride (DAPI) and penicillin/streptomycin were all obtained from Gibco (Thermo Fisher Scientific, Inc., Waltham, MA, USA). A cell counting kit-8 (CCK-8) was purchased from Dojindo Molecular Technologies, Inc. (Kumamoto, Japan; cat. no. CK04). An in situ cell apoptosis detection kit was purchased from Roche Diagnostics (Basel, Switzerland; cat no. 11684795910). Rabbit monoclonal antibodies for B-cell lymphoma 2 (Bcl-2)-associated X protein (Bax; cat no. 14796) and $\beta$-actin (cat no. 3700), and rabbit polyclonal antibodies for Bcl-2 (cat no. 2876), caspase-3 (cat no. 9662) and polyadenosine diphosphate-ribose polymerase (PARP; cat no. 9542) were purchased from Cell Signaling Technology, Inc. (Danvers, MA, USA). All other reagents used in the present study were of the highest available commercial grade and obtained from Sigma-Aldrich (Merck KGaA) unless stated otherwise.

Microsphere preparation and characterization. CS-CrmA microspheres were prepared according to an emulsion ionic cross-linking method modified from previously described methods (20). Briefly, 2 g CS was dispersed into $100 \mathrm{ml}$ acetic acid under vigorous stirring for $3 \mathrm{~h}$ at ambient temperature $\left(<20^{\circ} \mathrm{C}\right)$ to obtain transparent CS solution $(2 \% \mathrm{w} / \mathrm{v})$. Subsequently, microspheres were obtained via inotropic gelation between the positively charged amino groups of CS and the negatively charged amino groups of STPP and CrmA proteins. Under magnetic stirring with a thermostatic magnetic stirrer (MYP11-2; Shanghai Mei Yingpu Instrument Manufacturing Co., Ltd., Shanghai, China) at room temperature, $3.5 \mathrm{ml}$ of an aqueous solution of STPP $(0.06 \mathrm{mg} / \mathrm{ml})$ and $\mathrm{CrmA}(0.05 \mathrm{mg} / \mathrm{ml})$ was added to $3.5 \mathrm{ml} \mathrm{CS}$ solution [1\%, w/v, (pH 5.0)]. The reaction mixture was continuously stirred at room temperature for $10 \mathrm{~min}$ for complete stabilization of the system. Subsequently, the microspheres were transferred into Eppendorf tubes and isolated by centrifugation in a glycerol bed at $16,000 \mathrm{x} \mathrm{g}$ at $25^{\circ} \mathrm{C}$ for $30 \mathrm{~min}$. The supernatant was collected and the microspheres were then resuspended in ultrapure water by shaking on a vortex mixer (Ningbo Scientz Biotechnology Co., Ltd., Ningbo, China). Subsequently, the microspheres were centrifuged from the fixed volume of microsphere suspension under identical conditions without a glycerol bed. Following removal of the supernatant, CS-CrmA microspheres at the bottom of the vessel were collected. The CS microspheres were prepared using an identical method using a $0.9 \% \mathrm{NaCl}$ solution to replace $\mathrm{CrmA}$. Finally, the microspheres were fixed with $2 \%$ glutaraldehyde at room temperature for $30 \mathrm{~min}$ and freeze-dried, and then the Au-coated sizes and shapes of the microspheres were examined under a scanning electronic microscope (SEM; S-800; Hitachi, Ltd., Tokyo, Japan).

In vitro release profiles. Microspheres ( 30 mg) containing CrmA were placed in $201.5-\mathrm{ml}$ microcentrifuge tubes containing $1 \mathrm{ml}$ phosphate buffered saline (PBS). The microsphere suspension of four microcentrifuge tubes containing $\mathrm{CS}-\mathrm{CrmA}$ was agitated in a $37^{\circ} \mathrm{C}$ water bath at $60 \mathrm{x} \mathrm{g}$ for various time periods of up to 10 days $(0,1,2,3,4,5,6,7,8,9$ and 10 days) and the remaining 16 microcentrifuge tubes were stored at $4^{\circ} \mathrm{C}$ until they were agitated in order to perform the same procedure. Periodically, the microsphere suspension was centrifuged at $10,000 \mathrm{x} \mathrm{g}$ at $37^{\circ} \mathrm{C}$ for $15 \mathrm{~min}$ to harvest the supernatant for analysis of release $\mathrm{CrmA}$ following agitation, followed by resuspension of the microspheres in fresh PBS containing lysozyme (Thermo Fisher Scientific, Inc.; catalogue no. 89833). The CrmA concentration in the supernatant was assessed using an ELISA kit (cat no. CSB-EL004543RA; Cusabio Biotech Co., Ltd., Wuhan, China) according to the manufacturer's instructions.

Chondrocyte isolation and culture. A total of 20 7-day-old male Sprague-Dawley rats weighing 50 g were obtained from the Experimental Animal Center of Wuhan University (Wuhan, China). All experimental animals used in the experiments were individually maintained under standard conditions of controlled temperature $\left(22 \pm 1^{\circ} \mathrm{C}\right)$, lighting (12-h light/dark cycle) and humidity $(50 \pm 10 \%)$ with ad libitum access to food and water. Rats were euthanized by cervical dislocation following anesthesia with isoflurane $(3 \mathrm{ml} / \mathrm{kg}$; Shenzhen Reward Life Technology Co., Ltd., Shenzhen, China; catalogue no. R510-22; http://www.rwdmall.com/Shop/dongwumazui/mazuipeijian/mazuiji/186.html). The protocols for the animal experiment of the present study were in accordance with the recommendations and guidelines of the National Institutes of Health (Bethesda, MD, USA) and were approved by the Wuhan University Animal Care and Use Committee (Wuhan, China).

For the isolation of chondrocytes, cartilage was obtained from the knee joints of the rats and placed into PBS. Briefly, harvested cartilage was cut into small pieces, incubated in a $0.2 \%$ trypsin-containing solution and agitated at $80 \mathrm{x} \mathrm{g}$ for $2 \mathrm{~h}$ at $37^{\circ} \mathrm{C}$. Following digestion, the isolated chondrocytes were washed with DMEM and PBS twice, and then suspended in DMEM/F12 medium supplemented with 10\% FBS and $1 \%$ penicillin/streptomycin antibiotics $(1 \% \mathrm{v} / \mathrm{v})$ at $37^{\circ} \mathrm{C}$ in a humidified atmosphere with $5 \% \mathrm{CO}_{2}$. Cells were used at passage 0 or 1 to avoid dedifferentiation and maintained in a monolayer culture throughout the study. Additionally, cell viability was determined using a cell viability analyzer. Briefly, chondrocytes were seeded in 6- $\mathrm{cm}$ dishes at a density of $1 \times 10^{6}$ cells/well with a total volume of $2 \mathrm{ml}$ growth medium and cultured at $37^{\circ} \mathrm{C}$ in a humidified atmosphere with $5 \% \mathrm{CO}_{2}$ to $80 \%$ confluence. After washing with PBS, the cells were detached with $0.125 \%$ EDTA-trypsin and collected. The cell numbers were detected by the Vi-CELL cell viability analyzer (viability, >90\%; Beckman Coulter, Inc., Miami, FL, USA). 
Treatment. First-generation rat chondrocytes were maintained in a monolayer culture throughout the present study. After the cells were cultured in DMEM/F12 containing 10\% FBS and $1 \%$ penicillin/streptomycin and reached $70-80 \%$ confluence, the culture medium was removed and the cells were cultured in DMEM/F12 containing $2 \%$ FBS and $1 \%$ antibiotics at $37^{\circ} \mathrm{C}$ for $24 \mathrm{~h}$ after washing three times with PBS to avoid the influence of other cytokines. At the end of incubation, the FBS medium was removed, and the cells were incubated at $37^{\circ} \mathrm{C}$ with serum-free medium for $1 \mathrm{~h}$ before the onset of experimental treatments. The culture medium was replaced with DMEM/F12 supplemented with $10 \%$ FBS. Subsequently, IL-1 $\beta(10 \mathrm{ng} / \mathrm{ml})$ was added to the culture medium followed by incubation at $37^{\circ} \mathrm{C}$ for an additional $48 \mathrm{~h}$. Finally, chondrocytes were co-cultured with CS and CS-CrmA microspheres for a period of $4 \mathrm{~h}$. A negative control group was kept untreated except for replacement of the medium. A positive control group consisted of cells treated with $10 \mathrm{ng} / \mathrm{ml} \mathrm{IL}-1 \beta$ alone. Each group consisted of five independent samples from different rats, and each experiment was repeated 10 times.

Cell viability assay. The effect of microspheres on the cell viability of chondrocytes was assessed using a CCK-8 assay kit in accordance with the manufacturer's protocol. Chondrocytes were cultured at $37^{\circ} \mathrm{C}$ in 96 -well plates at a density of $1 \times 10^{4}$ cells/well in a total volume of $200 \mu 1$ growth medium (DMEM-F12 containing 2\% FBS). Following $48 \mathrm{~h}$ of co-culture with IL-1 $\beta(10 \mathrm{ng} / \mathrm{ml})$ and then $4 \mathrm{~h}$ of co-culture with CS and CS-CrmA microspheres, the culture medium was discarded and chondrocytes were washed twice with PBS. Next, $10 \mu \mathrm{l}$ CCK-8 reagent was added to each well and incubated at $37^{\circ} \mathrm{C}$ for $2 \mathrm{~h}$. The WST- 8 Tetrazolium salt in the reagent may be reduced to orange-yellow formazan by dehydrogenase, which is proportional to the number of viable cells. Furthermore, the absorbance at $450 \mathrm{~nm}$ was recorded using a microplate reader (Bio-Rad 680; Bio-Rad Laboratories, Inc., Hercules, CA, USA). A standard curve was designed using chondrocyte suspension with different dilution rates to calculate the viable cell numbers in each sample.

Quantification of NO. Nitrite levels in culture medium were assessed using Griess reaction, as previously described (21). To measure nitrite levels in the medium, $50 \mu 1$ sample aliquots were mixed with $50 \mu \mathrm{l}$ Griess reagent (catalogue no. G4410; Sigma-Aldrich; Merck KGaA) and then incubated at $37^{\circ} \mathrm{C}$ for $10 \mathrm{~min}$. After incubation, the absorbance was spectrophotometrically determined at the wavelength of $550 \mathrm{~nm}$ using a microplate reader (Bio-Rad 680; Bio-Rad Laboratories, Inc.). Nitrite concentrations were determined relative to a standard curve derived from increasing concentrations of sodium nitrite. Finally, the results were expressed as nmol/ml and were normalized against the control concentration.

Assay of $P G E_{2}$ concentrations. $\mathrm{PGE}_{2}$ levels were investigated using a commercially available ELISA kit (cat no. KGE004B; R\&D Systems, Inc., Minneapolis, MN, USA) according to the manufacturer's instructions. Furthermore, $\mathrm{PGE}_{2}$ concentrations were determined relative to a standard curve, and the results were expressed as $\mathrm{ng} / \mathrm{ml}$ and normalized against the control concentration.
Terminal deoxynucleotidyl transferase dUTP nick-end labeling (TUNEL) staining. An in situ cell apoptosis detection kit (catalogue no. 40306ES50; Shanghai Yeasen Biotechnology Co., Ltd., Shanghai, China) was used to detect cell apoptosis according to the manufacturer's instructions. Chondrocytes were seeded on cover slips in 24-well plates at a density of $1.25 \times 10^{5}$ cells/well in a total volume of $1 \mathrm{ml}$ growth medium (DMEM-F12 containing 2\% FBS). After implementing the above-described experimental design, the culture medium was removed from each well, and the chondrocytes were washed with PBS. Chondrocytes were stained with DAPI at $37^{\circ} \mathrm{C}$ for $30 \mathrm{~min}$. Additionally, apoptotic chondrocytes were recognized with dual TUNEL and DAPI staining. After the final wash with PBS, cells were observed under an inverted fluorescence microscope, and apoptosis signals were counted manually. Furthermore, images were randomly selected from three fields of each specimen and the stained cells were counted under x200 magnification. A control group was kept untreated with the exception of replacing the medium without IL-1 $\beta, \mathrm{CS}$ and CS-CrmA microspheres. The rate of TUNEL-positive cells in each field was calculated.

RNA extraction and reverse transcription-quantitative polymerase chain reaction (RT-qPCR). TRIzol and chloroform reagents (Sigma-Aldrich, Merck KGaA) were used to extract the total RNA from chondrocytes according to the manufacturer's instructions. Briefly, $2 \mathrm{ml}$ TRIzol reagent was added to split the chondrocytes for $20 \mathrm{~min}$, and the sample was then transferred into a $2 \mathrm{ml}$ Eppendorf tube. Subsequently, $400 \mu \mathrm{l}$ chloroform was added. The tube was vigorously agitated for $30 \mathrm{sec}$ and allowed to stand for $15 \mathrm{~min}$. The sample was then centrifuged at $13,000 \mathrm{x} \mathrm{g}$ for $15 \mathrm{~min}$ at $4^{\circ} \mathrm{C}$. The supernatant from the final extraction step was transferred to a clean $2 \mathrm{ml}$ Eppendorf tube and the RNA precipitated with $500 \mu 1$ isopropanol at $-20^{\circ} \mathrm{C}$ for $2 \mathrm{~h}$. Precipitated RNA was collected by centrifugation at $13,000 \mathrm{x}$ g for $15 \mathrm{~min}$ at $4^{\circ} \mathrm{C}$, and the pellet was washed with $1 \mathrm{ml} 75 \%$ ice-cold ethanol. The RNA pellet was then resuspended in $20 \mu 1$ nuclease-free water and the two duplicate tubes were combined. The RNA concentration was determined using a spectrophotometer (Biolab ND-1000; Thermo Fisher Scientific, Inc.) at $260 \mathrm{~nm}$, and the purity was assessed by measuring the A260/A280 ratio. Purified RNA with an A260/A280 ratio between 1.8 and 2.0 was used in the present study.

cDNA was synthesized from RNA using a PrimeScript reverse transcriptase kit (cat. no. AB-1455/A; Fermentas; Thermo Fisher Scientific, Inc.) according to the manufacturer's instructions. The total extracted RNA was diluted to the final concentration of $1 \mu \mathrm{g} / \mu \mathrm{l}$. RT-qPCR was performed using a $20 \mu \mathrm{l}$ reaction volume containing $10 \mu \mathrm{l}$ SYBRpremix Ex Taq II (Takara Bio, Inc., Otsu, Japan), $1 \mu$ l RNase-free DNase I (catalogue no. EN0521; Fermentas; Thermo Fisher Scientific, Inc.), $0.4 \mu \mathrm{l}$ ROX Reference Dye II, $0.8 \mu \mathrm{l}$ each forward and reverse primer, $2 \mu \mathrm{l}$ cDNA and $6 \mu 1$ nuclease-free water. Reactions were run on a 7500 Real Time PCR system (Thermo Fisher Scientific, Inc.) for 45 consisting of the following steps: Initial denaturation at $95^{\circ} \mathrm{C}$ for $5 \mathrm{~min}$, followed by a set cycle of denaturation at $94^{\circ} \mathrm{C}$ for $10 \mathrm{sec}$, and different annealing temperatures for each pair of primers (ranging between 53 and $62^{\circ} \mathrm{C}$ ) for $10 \mathrm{sec}$, extension at $72^{\circ} \mathrm{C}$ for $28 \mathrm{sec}$, and a 
Table I. Sequences of primers for the reverse transcription quantitative polymerase chain reaction experiments.

\begin{tabular}{|c|c|c|c|}
\hline Gene & Direction & Sequence $5^{\prime} \rightarrow 3^{\prime}$ & Size (bp) \\
\hline \multirow[t]{2}{*}{ iNOS } & $\mathrm{F}$ & ACCAGTACGTTTGGCAATGG & 70 \\
\hline & $\mathrm{R}$ & TCAGCATGAAGAGCGATTTCT & \\
\hline \multirow[t]{2}{*}{ COX-2 } & $\mathrm{F}$ & CTTACAATGCTGACTATGGCTAC & 242 \\
\hline & $\mathrm{R}$ & AAACTGATGCGTGAAGTGCTG & \\
\hline \multirow[t]{2}{*}{$\mathrm{Bcl}-2$} & $\mathrm{~F}$ & CCACCAAGAAAGCAGGAAACC & 177 \\
\hline & $\mathrm{R}$ & GGCAGGATAGCAGCACAGG & \\
\hline \multirow[t]{2}{*}{$\operatorname{Bax}$} & $\mathrm{F}$ & CAGATGTGGTCTATAATGC & 110 \\
\hline & $\mathrm{R}$ & CTAATCAAGTCAAGGTCAC & \\
\hline \multirow[t]{2}{*}{ Caspase-3 } & $\mathrm{F}$ & CATGGAAGCGAATCAATGGACT & 139 \\
\hline & $\mathrm{R}$ & CTGTACCAGACCGAGATGTCA & \\
\hline \multirow[t]{2}{*}{ PARP } & $\mathrm{F}$ & TCTTTGATGTGGAAAGTATGAAGAA & 64 \\
\hline & $\mathrm{R}$ & GGCATCTTCTGAAGGTCGAT & \\
\hline \multirow[t]{2}{*}{$\beta$-actin } & $\mathrm{F}$ & CGTTGACATCCGTAAAGAC & 201 \\
\hline & $\mathrm{R}$ & TGGAAGGTGGACAGTGAG & \\
\hline
\end{tabular}

iNOS, inducible nitric oxide synthase; COX-2, cyclooxygenase-2; Bcl-2, B-cell lymphoma 2; Bax, Bcl-2-associated X protein; PARP, poly ADP ribose polymerase; $\mathrm{F}$, forward; $\mathrm{R}$, reverse.

final elongation at $72^{\circ} \mathrm{C}$ for $5 \mathrm{~min}$. The generation of specific PCR products were subjected to melting curve analysis, and gene expression was normalized to the expression of the housekeeping gene, $\beta$-actin, and expressed as the fold ratio compared with the control according to previously described method by Cheng et al (2). Sequences of primers used (Takara Bio, Inc.) are presented in Table I.

Western blot analysis. Protein was extracted from harvested chondrocytes using a cell extraction buffer (cat no. FNN0011; Fermentas, Thermo Fisher Scientific, Inc.) following the manufacturer's protocol. The protein concentrations were determined using the bicinchoninic acid protein assay kit (cat no. 23225; Fermentas, Thermo Fisher Scientific, Inc.). After chondrocytes were treated according to the experimental design, the culture medium was discarded and chondrocytes were washed twice with precooled PBS. The chondrocytes were then collected using a cell scraper and transferred into a $1.5 \mathrm{ml}$ Eppendorf tube, a cell extraction buffer (catalogue no. FNN0011; Fermentas, Thermo Fisher Scientific, Inc.) containing protease and phosphatase inhibitors (catalogue no. 78440; Fermentas, Thermo Fisher Scientific, Inc.) was added and placed on ice for $30 \mathrm{~min}$. The lysate was collected and centrifuged at $12,000 \mathrm{x}$ g for $20 \mathrm{~min}$ at $4^{\circ} \mathrm{C}$. Each well in the $10 \%$ SDS-PAGE was loaded with $20 \mu \mathrm{l}$ protein. After adjusting for equal amounts of total protein, protein mixtures were separated by SDS-PAGE and transferred onto nitrocellulose membranes (Fermentas, Thermo Fisher Scientific, Inc.). Following the transfer, non-specific binding sites of the membranes were blocked for $1 \mathrm{~h}$ at room temperature in PBS (pH 7.4) containing 5\% non-fat dry milk, and then incubated overnight at $4^{\circ} \mathrm{C}$ with the following antibodies (all dilutions, 1:1,000): anti-Bcl-2, anti-Bax, anti-caspase-3 and anti-PARP. Anti-Bcl-2 did not cross-react with Bcl-2 $\beta$ or other Bcl-2 family members, Bax recognized endogenous levels of total
Bax protein in rodent samples, caspase-3 detected endogenous levels of caspase-3 resulting from cleavage and PARP did not cross-react with related proteins or other PARP isoforms.

In order to control protein loading, the membranes were probed with an anti- $\beta$-actin antibody (dilution, 1:1,000; catalogue no. 3700; Cell Signaling Technology, Inc.). Subsequently, the membranes were washed three times with TBST $[10 \mathrm{mM}$ Tris- $\mathrm{HCl}$, (pH 7.4), $100 \mathrm{mM} \mathrm{NaCl}$ and 0.2\% Tween-20] and incubated with horseradish peroxidase-conjugated secondary antibodies (goat anti-rabbit immunoglobulin G; catalogue no. 7075; dilution, 1:1,000; Cell Signaling Technology, Inc.), followed by visualization using the enhanced chemiluminescence kit (cat. no. 35085; Fermentas; Thermo Fisher Scientific, Inc.). The results were scanned using a gel imaging system (Geldoc-It 310; UVP, LLC., Upland, CA, USA), and densitometry measurements were performed with Image Lab version 4.1 software (Bio-Rad Laboratories, Inc.).

Statistical analysis. Values were expressed as the mean \pm standard deviation and statistical analyses were performed using SPSS software, version 19.0 (IBM Corp., Armonk, NY, USA). Each experimental condition was performed in triplicate wells, and the mean from each culture replicate was calculated and combined as one value for analysis. Significant differences among the mean values of multiple groups were evaluated with analysis of variance followed by Student-Newman-Keuls' method. A two-sided $\mathrm{P}<0.05$ was considered to indicate a statistically significant difference.

\section{Results}

Characterization of microspheres. Microsphere morphology was observed via SEM (Fig. 1). The CS-CrmA microspheres, which were fabricated using the emulsification method in the 

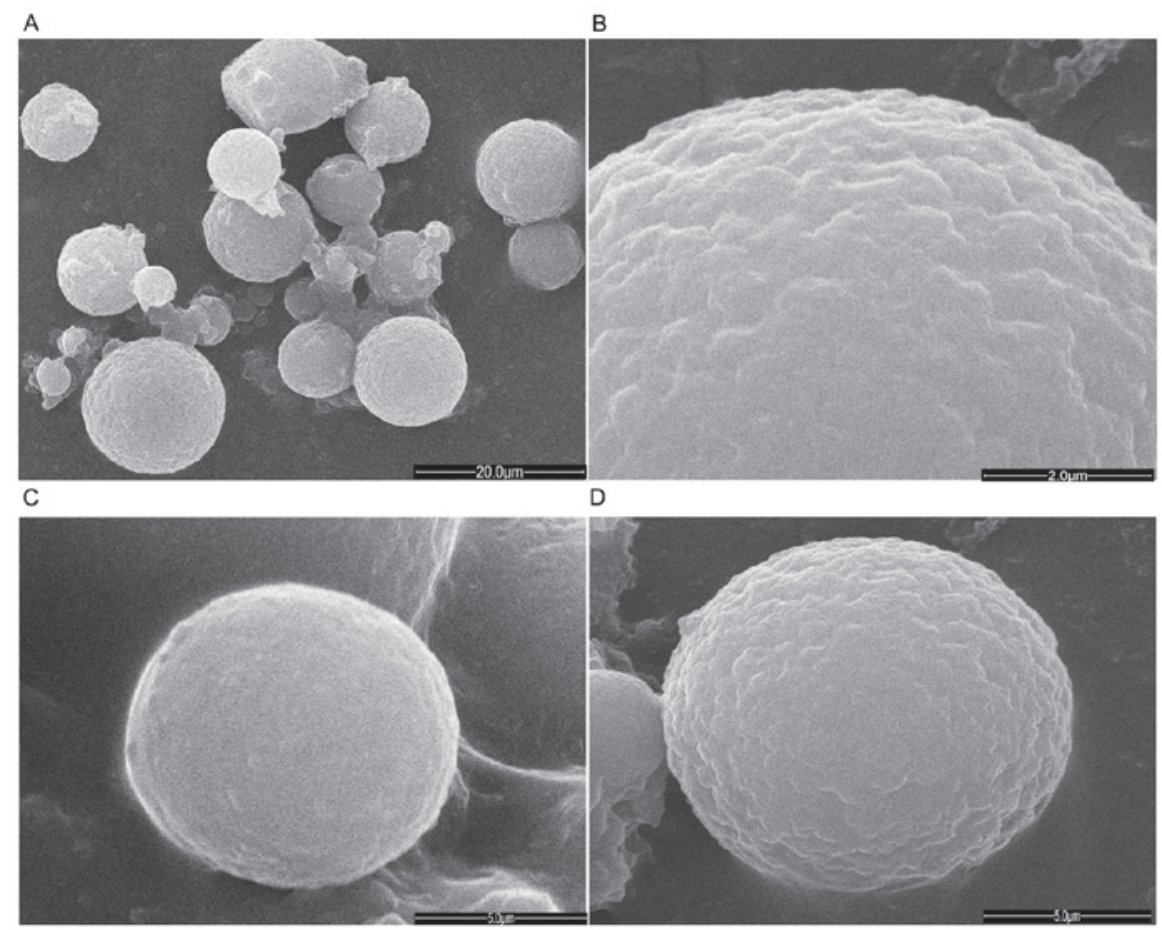

Figure 1. Characterization of microspheres by scanning electron microscopy. (A) The microspheres were uniformly distributed and ranged in size from 8-17 $\mu \mathrm{m}$ (magnification, $\mathrm{x} 2,000$ ). (B) The microspheres were spherical and the surfaces were almost smooth (magnification, $\mathrm{x} 2,000$ ). With regard to the variation in composition and structure, there was a slight decrease in the microsphere size in (C) chitosan (magnification, x12,000) compared with (D) chitosan-cytokine response modifier A microspheres (magnification x12,000).

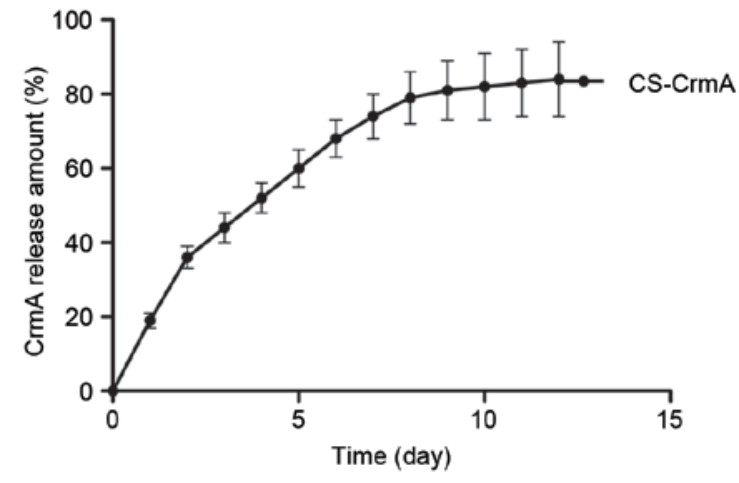

Figure 2. Release kinetics of CrmA microspheres. Values are expressed as the mean \pm standard deviation. CS, chitosan; CrmA, cytokine response modifier A.

presence of STPP, were spherical in shape with smooth surfaces The resulting microspheres were spherical and ranged in size from 8-17 $\mu \mathrm{m}$ (Fig. 1A). Furthermore, the microsphere surface appeared to be porous and relatively smooth (Fig. 1B). With regard to the variation in composition and structure, a slight decrease in microsphere size was observed in CS (Fig. 1C) compared with CS-CrmA microspheres (Fig. 1D).

In vitro release profiles. The release kinetics of $\mathrm{CrmA}$ from CS-CrmA microspheres are presented in Fig. 2. The CrmA release kinetics were monitored for 10 days and characterized by an initial burst release, which was gradually reduced to a linear release. The CrmA from CS-CrmA microspheres was released slowly. The final release rate was $\sim 82 \%$ in the CS-CrmA microspheres at 10 days of incubation.

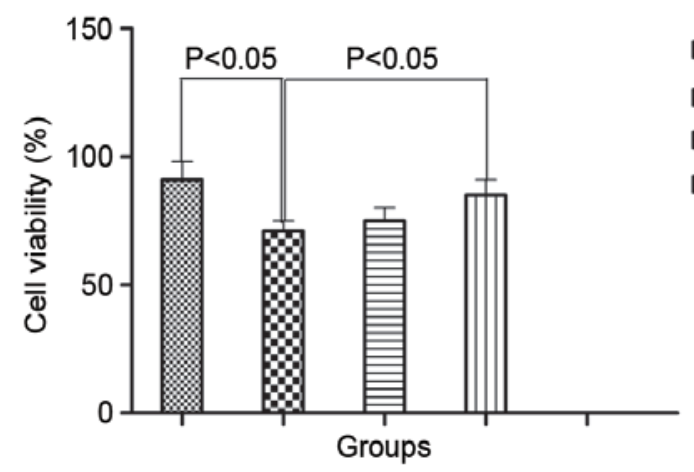

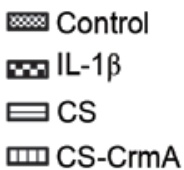

Figure 3. CS-mediated release of CrmA attenuates IL-1 $\beta$-induced reduction of chondrocyte viability. Values are expressed as the mean \pm standard deviation. IL-1 $\beta$, interleukin-1 $\beta$; CS, chitosan; CrmA, cytokine response modifier A.

CrmA-releasing microspheres attenuated IL-1 $\beta$-mediated reduction in cell viability. Cell viability in the various experimental groups is presented in Fig. 3. The cell viability of the IL-1 $\beta$ group was $71 \pm 4 \%$, which was significantly lower than $91 \pm 7 \%$ of the control group $(\mathrm{P}<0.05)$. Slight but not marked differences in cell viability were also observed between the IL-1ß $(71 \pm 4 \%)$ and CS $(75 \pm 5 \%)$ groups. However, co-treatment with CS-CrmA significantly increased the cell viability to $85 \pm 6 \%(\mathrm{P}<0.05$ vs. IL-1 $\beta)$.

CrmA-releasing microspheres attenuate $I L-1 \beta$-induced gene expression of $i N O S$ and $C O X-2$ and release of $N O$ and $P G E_{2}$ by chondrocytes. The gene expression of iNOS and COX-2 and the concentrations of $\mathrm{NO}$ and $\mathrm{PGE}_{2}$ in the various experimental groups are depicted in Fig. 4A-D. Stimulation 


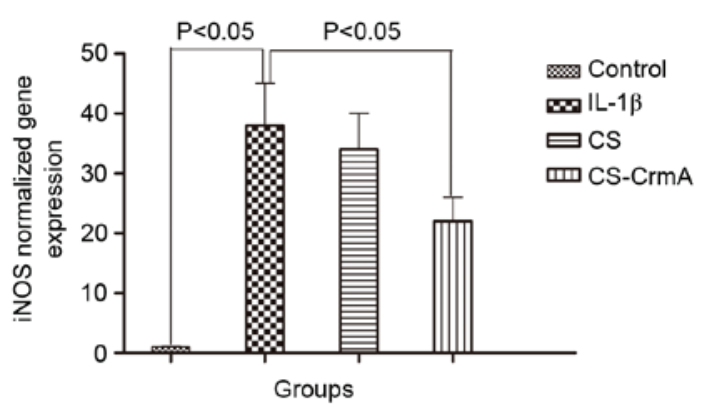

c

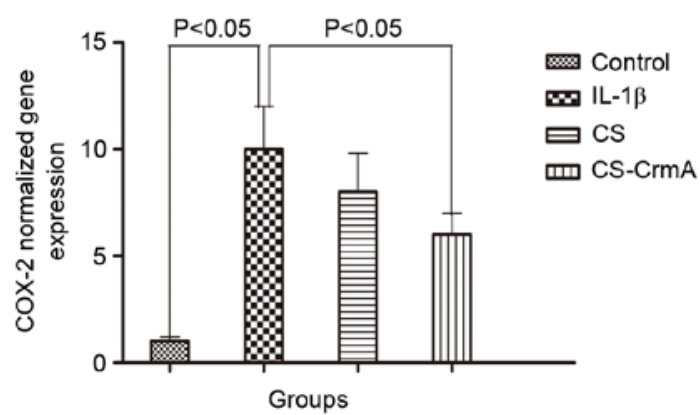

B

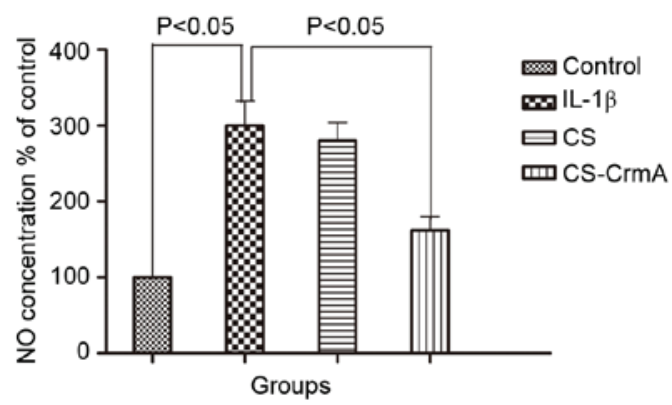

D

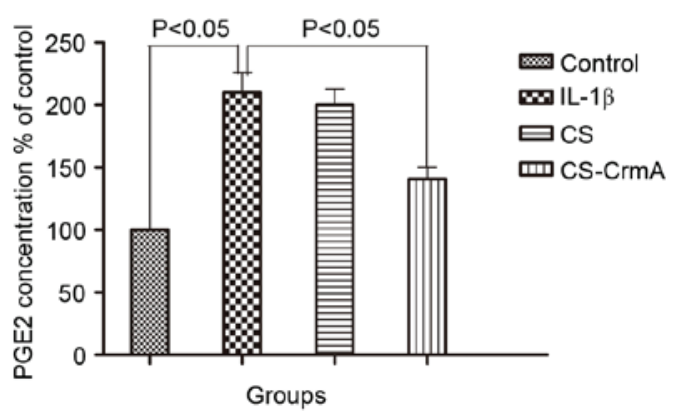

Figure 4. Effect of microspheres on IL-1 $\beta$-induced gene expression of (A) iNOS and (B) COX-2 and the production of (C) NO and (D) PGE 2 . The normalized levels of gene expression were expressed as the ratios of the copy number of the mRNA of the targeted genes and of $\beta$-actin cDNA. Culture media were analyzed for nitrite concentration on behalf of NO production. Values were expressed as the mean \pm standard deviation. iNOS, inducible nitric oxide synthase; IL-1 $\beta$, interleukin-1 $\beta$; CS, chitosan; CrmA, cytokine response modifier A; NO, nitric oxide; COX-2, cyclooxygenase-2; PGE 2 , prostaglandin E2.

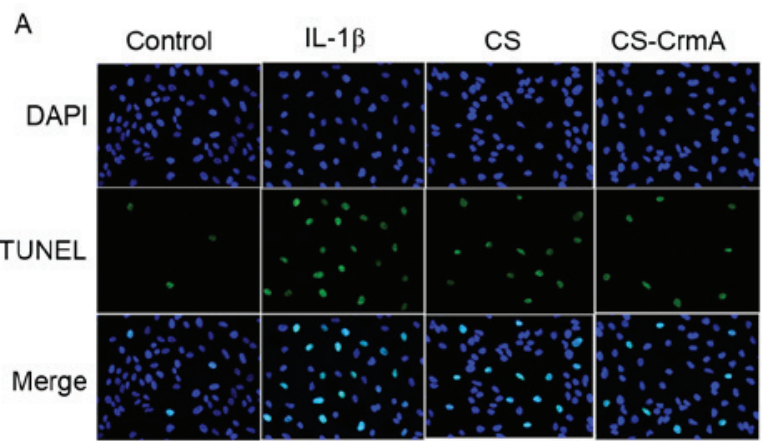

B

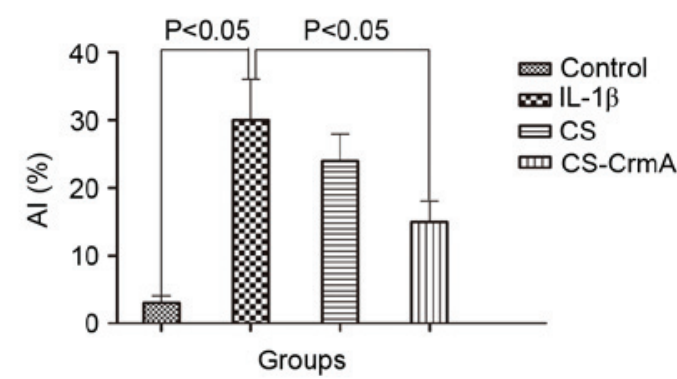

Figure 5. CS-mediated release of CrmA reduces IL-1 $\beta$-induced chondrocyte apoptosis. (A) Apoptosis in various treatment groups was assessed by TUNEL staining and fluorescence microscopic analysis (magnification, x200). Nuclei were counter-stained with DAPI. (B) AIs were obtained by quantification of apoptosis. Values were expressed as the mean \pm standard deviation. DAPI, 4',6-diamidino-2-phenylindole; TUNEL, terminal deoxynucleotidyl transferase dUTP nick end labeling; IL-1 $\beta$, interleukin-1 $\beta$; CS, chitosan; CrmA, cytokine response modifier A; AI, apoptotic index.

with IL-1 $\beta(10 \mathrm{ng} / \mathrm{ml})$ for $48 \mathrm{~h}$ led to a 38 -fold increase in iNOS expression and a 3.0 -fold increase in NO production in the supernatant, compared with controls (both $\mathrm{P}<0.05$ ). Furthermore, CS microspheres slightly but not markedly decreased this iNOS expression and NO production, whereas CS-CrmA microspheres significantly decreased the iNOS expression and NO production, compared with the IL-1 $\beta$ group (both $\mathrm{P}<0.05$ ). Similar patterns were observed for the COX-2 expression and $\mathrm{PGE}_{2}$ concentration in the supernatant.

CrmA-releasing microspheres reduce $I L-1 \beta$-induced chondrocyte apoptosis. TUNEL staining of chondrocytes in the different treatment groups for the detection of apoptosis and apoptotic index is shown in Fig. 5. The percentage of TUNEL-positive cells in the IL- $1 \beta$ group was $30 \pm 6 \%$, whereas that in the untreated control group was significantly lower at only $3 \pm 1 \%(\mathrm{P}<0.05)$. Compared with the IL-1 $\beta$ group, co-treatment with CS slightly but not markedly decreased the apoptotic rate of the chondrocytes $(24 \pm 4$ vs. $30 \pm 6 \%)$. However, following co-culture with CS-CrmA microspheres, the percentage of TUNEL-positive cells was $15 \pm 3 \%$, which was significantly lower than that in the IL-1 $\beta$ group $(\mathrm{P}<0.05)$.

CrmA-releasing microspheres attenuate $I L-1 \beta$-induced apoptosis signaling. The gene expressions of Bcl-2, Bax, caspase- 3 and PARP in various experimental groups are presented in Fig. 6A-D, respectively. Stimulation with IL-1 $\beta$ $(10 \mathrm{ng} / \mathrm{ml})$ led to a significant decrease of Bcl-2 expression and a significant increase of Bax, caspase- 3 and PARP expression compared with those in the control group (all $\mathrm{P}<0.05$ ). Slight but not marked differences in the gene expression of Bcl-2, Bax, caspase-3 and PARP were observed between the IL-1 $\beta$ and CS groups ( $>>0.05)$. However, the expression of Bcl-2 was significantly increased following co-treatment with 
A

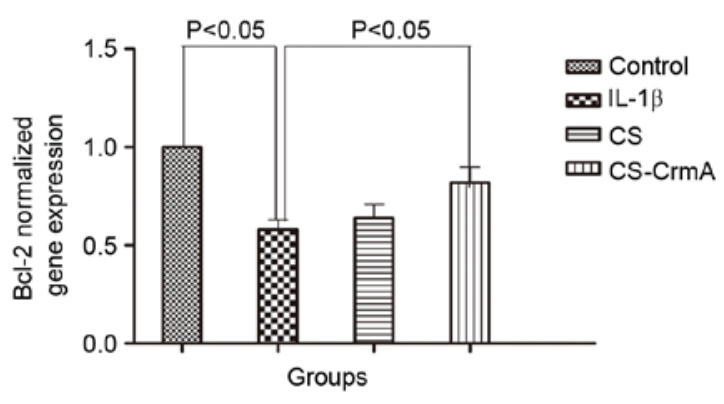

C

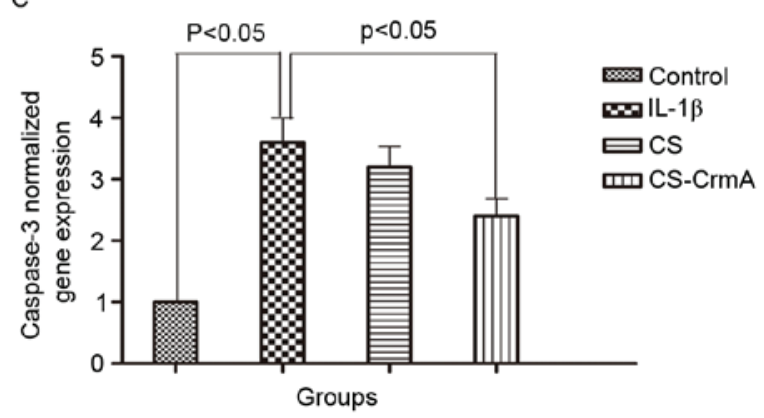

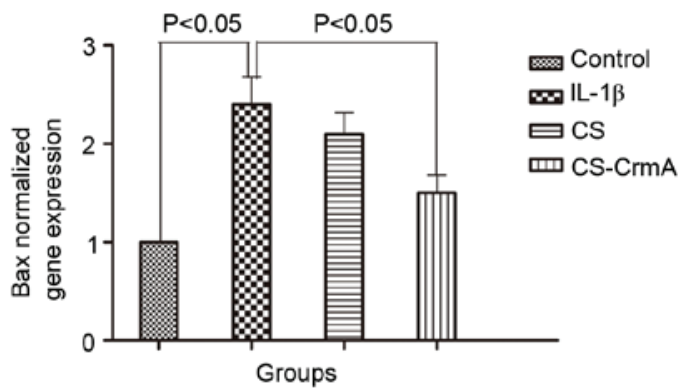

D

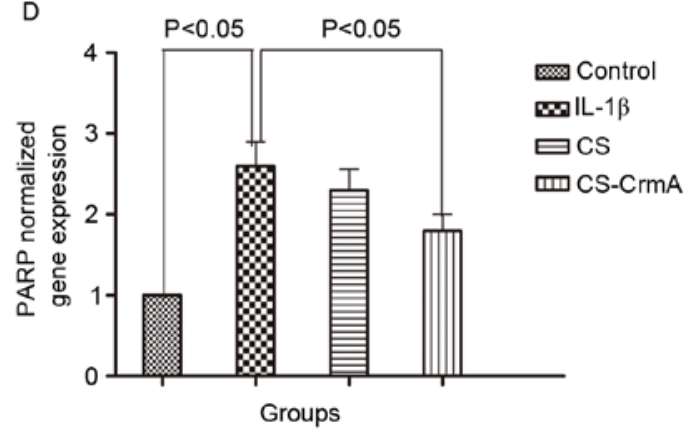

Figure 6. Effect of microspheres on the relative expression of (A) Bcl-2, (B) Bax, (C) caspase-3 and (D) PARP mRNA. The normalized gene expression levels were expressed as ratios of the copy number of the mRNA of the targeted genes and that of $\beta$-actin cDNA. Values were expressed as the mean \pm standard deviation. Bcl-2, B-cell lymphoma 2; IL-1 $\beta$, interleukin-1 $\beta$; CS, chitosan; CrmA, cytokine response modifier A; Bax, Bcl-2-associated X protein; PARP, poly adenosine diphosphate-ribose polymerase.

CS-CrmA microspheres $(\mathrm{P}<0.05)$, whereas the expressions of Bax, caspase-3 and PARP were significantly decreased (all $\mathrm{P}<0.05)$ following co-treatment with $\mathrm{CS}-\mathrm{CrmA}$ microspheres compared with those in the IL-1 $\beta$ group.

The protein levels and relative intensities of $\mathrm{Bcl}-2$, Bax, caspase- 3 and PARP in the experimental groups are presented in Fig. 7A-D, respectively. The results revealed similar trends to the mRNA levels of the respective proteins. Compared with the control group, the gene expression of $\mathrm{Bcl}-2$ was significantly reduced by IL- $1 \beta$, whereas the gene expressions of Bax, caspase-3 and PARP were significantly increased by IL-1 $\beta$ (all $\mathrm{P}<0.05$ ). Slight but not marked differences were observed in the expression of Bcl-2, Bax, caspase-3 and PARP between the IL-1 $\beta$ and CS groups. However, the expression of Bcl-2 was significantly increased in the CS-CrmA group compared with that in the IL-1 $\beta$ group $(\mathrm{P}<0.05)$, whereas the expressions of Bax, caspase-3 and PARP were significantly decreased (all $\mathrm{P}<0.05)$.

\section{Discussion}

OA is a degenerative disease that is characterized by the progressive loss of articular cartilage and destruction of cartilage matrix (22). It is widely accepted that IL-1 $\beta$ is important in promoting OA lesions by leading to the production and accumulation of high levels of pro-inflammatory cytokines and by triggering apoptosis in chondrocytes (22). Therefore, using CrmA to inhibit the IL-1 $\beta$ pathway may prevent inflammation and apoptosis in chondrocytes.

In the present study, it was observed that CS was able to enhance the ability of the microspheres to interact with the chondrocytes and the controlled-release of the loaded drug.
Additionally, the release rate of CrmA from the CS-CrmA microspheres was slow. These results were further confirmed by the microsphere structure and release kinetics of proteins from the microspheres.

In the present study, CS slightly but not markedly increased the cell viability of chondrocytes. In a previous study, administration of CS solution into the murine knee joint led to a significant increase in the density of newly formed chondrocytes, which indicated that it was able to facilitate the healing of the cartilage (19). Furthermore, it was revealed that the percentage of viable cells in the CS-CrmA group was significantly higher than that in the IL- $1 \beta$ group, a finding that was in accordance with that of a recent study by the present authors (7).

It has been demonstrated that iNOS is able to catalyze oxidation to generate amounts of NO within the joint fluid of OA chondrocytes, leading to cartilage destruction (23). COX-2 is a rate-limiting enzyme for the generation of $\mathrm{PGE}_{2}$ metabolites, which are important mediators of inflammation and the anabolic/catabolic process associated with OA (24). In the present study, CS slightly but not markedly decreased the gene expression of iNOS and COX-2 and the production of $\mathrm{NO}$ and $\mathrm{PGE}_{2}$. However, CS-CrmA significantly inhibited the gene expression of iNOS and COX-2 and the production of $\mathrm{NO}$ and $\mathrm{PGE}_{2}$. These observations indicate that IL-1 $\beta$-induced uncontrolled inflammation in chondrocytes may be attenuated by CS-CrmA microspheres. Therefore, it is suggested that CS-CrmA microspheres may act as anti-inflammatory agents similar to NSAIDs, which have been indicated to ameliorate OA symptoms by inhibiting the production of $\mathrm{PGE}_{2}$. However, it is likely that the control-released action had a longer duration in the presence of CS. This may have been due to the 

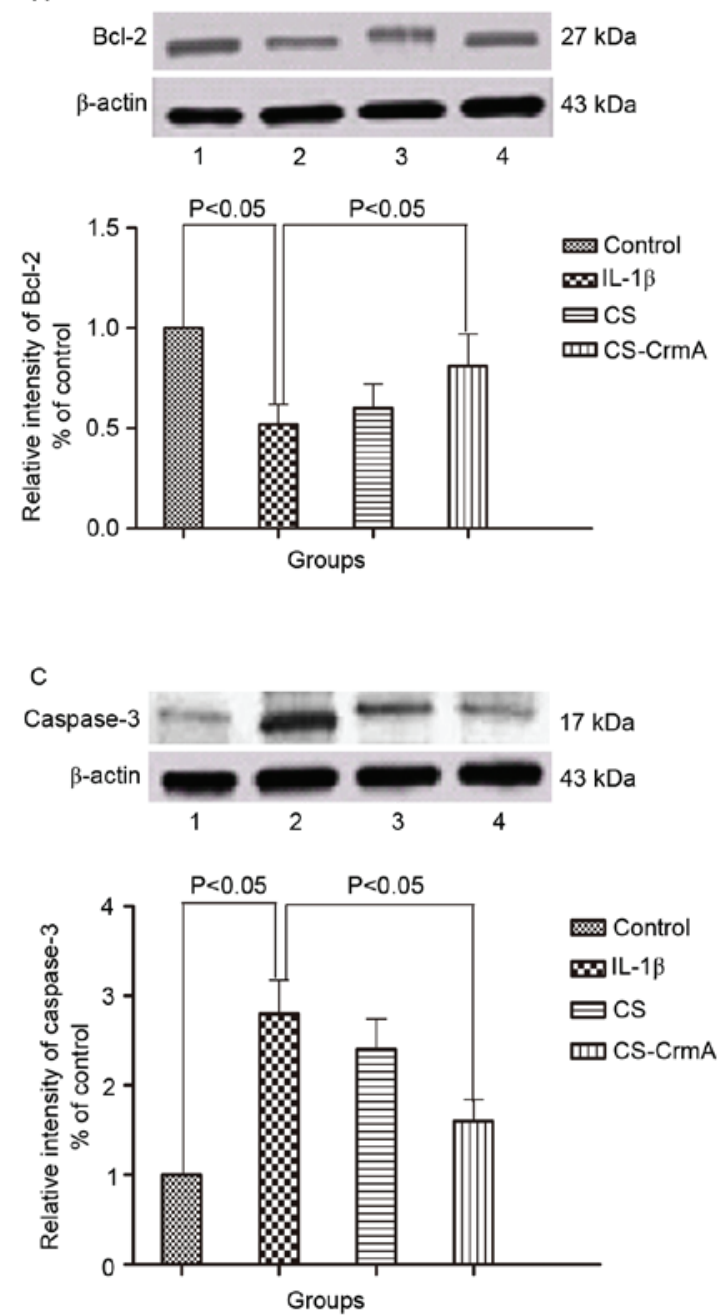
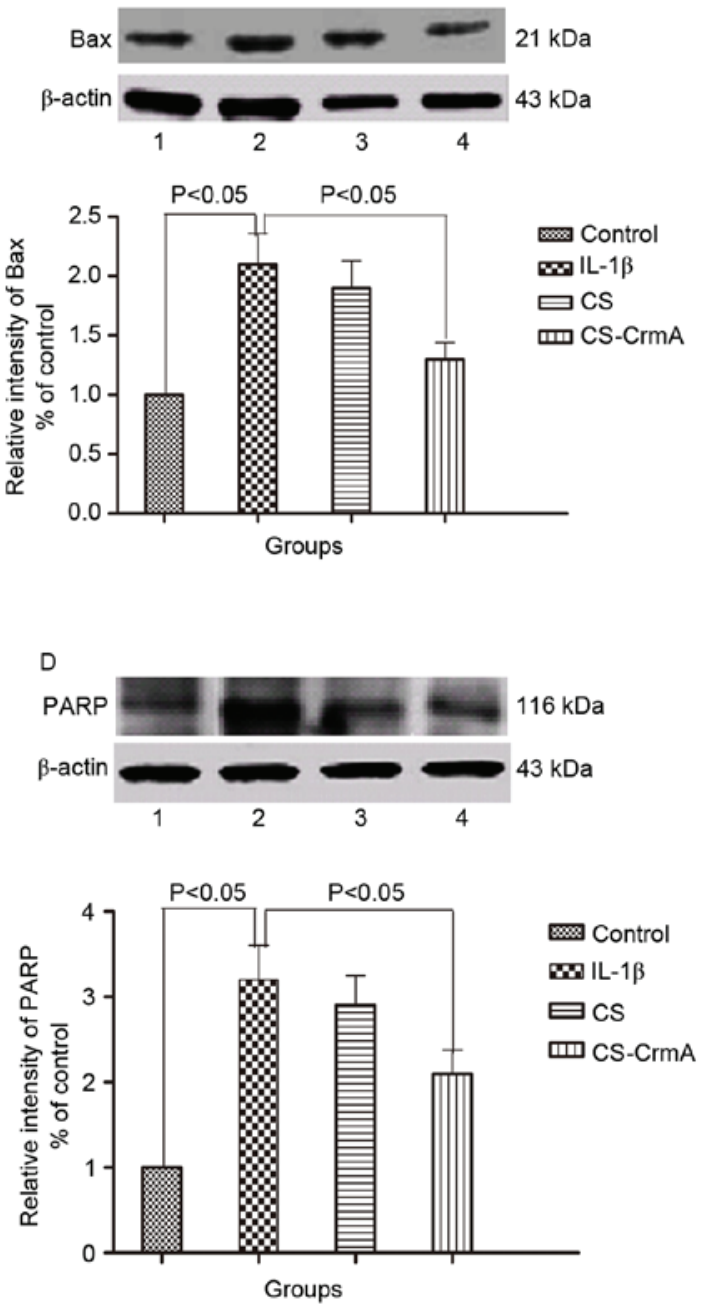

Figure 7. Western blot analysis and relative intensities of (A) Bcl-2, (B) Bax, (C) caspase-3 and (D) PARP. Lanes: 1, control; 2 , IL-1 $\beta$; 3 , CS; 4, CS-CrmA. Protein levels were normalized to $\beta$-actin. Values were expressed as the mean \pm standard deviation. Bcl-2, B-cell lymphoma 2; Bax, Bcl-2-associated X protein; PARP, poly adenosine diphosphate-ribose polymerase; IL-1 $\beta$, interleukin-1 $\beta$; CS, chitosan; CrmA, cytokine response modifier A.

capacity of the polymer to interact with the negatively charged cell surfaces.

Apoptosis is a highly-regulated, active process of cell death that is associated with the development of human and animal OA (25). In the present study, it was revealed that CS slightly but not markedly decreased the percentage of apoptotic chondrocytes. Additionally, the percentage of apoptotic cells was significantly decreased in the CS-CrmA group. It can be inferred that CS-CrmA microspheres are able to suppress chondrocyte apoptosis in an IL-1 $\beta$-induced OA model.

Bcl-2 family proteins can be readily heterodimerized by protein-protein interactions between pro- and anti-apoptotic Bcl-2 family members, which determine whether cell survival or the apoptosis signal proceeds $(26,27)$. The activation of caspase- 3 is the most important pathway during the apoptotic process, which then induces hydrolysis of nucleic acids and cytoskeletal proteins $(28,29)$. During apoptotic cell death, activated effector caspases cleave multiple cellular substrates, including the DNA repair enzyme PARP in chondrocytes (30). The present study demonstrated that CS slightly but not markedly increased the expression of Bcl-2 and decreased the expressions of Bax, caspase- 3 and PARP in transcriptional and translational levels than those in the IL-1 $\beta$ group. Nevertheless, the CS-CrmA microspheres had significantly increased the expression of Bcl-2 and decreased the expressions of Bax, caspase-3 and PARP in the transcriptional and translational levels than compared with those in the IL-1 $\beta$ group. These results further confirmed the previous results of cell viability and apoptosis assay.

In conclusion, the suppression of inflammation and apoptosis within the joint may also be one important mechanism of the clinical activity of microspheres in OA treatment. Therefore, the present results suggest that the sustained release of CrmA from CS-CrmA microspheres may prevent IL-1 $\beta$-induced inflammation and apoptosis in chondrocytes. This suggests that the use of CS-CrmA microspheres as CrmA carriers with controlled and prolonged release properties for the treatment of $\mathrm{OA}$ is promising.

\section{Acknowledgements}

The authors would like to thank the Department of Orthopedics in The Renmin Hospital of Wuhan University. The present study was supported by the National Natural Science 
Foundation of China (grant no. 81071494), the National Natural Science Foundation of China (grant no. 81501921), the Hubei Provincial Science and Technology Support Program of China (grant no. 2015BCA316), the Central University Basic Scientific Research Business Expenses Special Funds Project of China (grant no. 2042015kf1014), the Health and Family Planning Research Project of Hubei Province of China (grant no. WJ2015MB024) and the Natural Science Foundation of Hubei Province of China (grant no. 2014CFB207).

\section{References}

1. Ying X, Peng L, Chen H, Shen Y, Yu K and Cheng S: Cordycepin prevented IL- $\beta$-induced expression of inflammatory mediators in human osteoarthritis chondrocytes. Int Orthop 38: 1519-1526, 2014.

2. Cheng W, Wu D, Zuo Q, Wang Z and Fan W: Ginsenoside Rb1 prevents interleukin-1 beta induced inflammation and apoptosis in human articular chondrocytes. Int Orthop 37: 2065-2070, 2013.

3. ElMansouriFE,Nebbaki SS, Kapoor M,AfifH,Martel-PelletierJ, Pelletier JP, Benderdour M and Fahmi H: Lysine-specific demethylase 1-mediated demethylation of histone $\mathrm{H} 3$ lysine 9 contributes to interleukin $1 \beta$-induced microsomal prostaglandin E synthase 1 expression in human osteoarthritic chondrocytes. Arthritis Res Ther 16: R113, 2014.

4. Stratz C, Anakwue J, Bhatia H, Pitz S and Fiebich BL: Anti-inflammatory effects of 5-HT3 receptor antagonists in interleukin-lbeta stimulated primary human chondrocytes. Int Immunopharmacol 22: 160-166, 2014

5. López-Armada MJ, Caramés B, Lires-Deán M, Cillero-Pastor B, Ruiz-Romero C, Galdo F and Blanco FJ: Cytokines, tumor necrosis factor-alpha and interleukin-1beta, differentially regulate apoptosis in osteoarthritis cultured human chondrocytes. Osteoarthritis Cartilage 14: 660-669, 2006.

6. Zhou PH, Liu SQ and Peng H: The effect of hyaluronic acid on IL-1beta-induced chondrocyte apoptosis in a rat model of osteoarthritis. J Orthop Res 26: 1643-1648, 2008.

7. Ma BL, Zhou PH, Xie T, Shi L, Qiu B and Wang Q: Inhibition of interleukin-1beta-stimulated dedifferentiation of chondrocytes via controlled release of CrmA from hyaluronic acid-chitosan microspheres. BMC Musculoskelet Disord 16: 61, 2015.

8. D'Lima D, Hermida J, Hashimoto S, Colwell C and Lotz M: Caspase inhibitors reduce severity of cartilage lesions in experimental osteoarthritis. Arthritis Rheum 54: 1814-1821, 2006.

9. Joosten LA, Netea MG, Fantuzzi G, Koenders MI, Helsen MM, Sparrer H, Pham CT, van der Meer JW, Dinarello CA and van den Berg WB: Inflammatory arthritis in caspase 1 gene-deficient mice: Contribution of proteinase 3 to caspase 1-independent production of bioactive interleukin-1beta. Arthritis Rheum 60: 3651-3662, 2009.

10. Dash M, Chiellini F, Ottenbrite RM and Chiellini E: Chitosan-A versatile semi-synthetic polymer in biomedical applications. Prog Polym Sci 36: 981-1014, 2011.

11. Sobol M, Bartkowiak A, de Haan B and de Vos P: Cytotoxicity study of novel water-soluble chitosan derivatives applied as membrane material of alginate microcapsules. J Biomed Mater Res A 101: 1907-1914, 2013.

12. Lih E, Lee JS, Park KM and Park KD: Rapidly curable chitosan-PEG hydrogels as tissue adhesives for hemostasis and wound healing. Acta Biomater 8: 3261-3269, 2012.

13. Chang SH, Hsiao YW and Lin HY: Low-frequency electromagnetic field exposure accelerates chondrocytic phenotype expression on chitosan substrate. Orthopedics 34: 20, 2011.
14. Sarvaiya J and Agrawal YK: Chitosan as a suitable nanocarrier material for anti-Alzheimer drug delivery. Int J Biol Macromol 72: 454-465, 2015.

15. David KI, Jaidev LR, Sethuraman S and Krishnan UM: Dual drug loaded chitosan nanoparticles-sugar-coated arsenal against pancreatic cancer. Colloids Surf B Biointerfaces 135: 689-698, 2015.

16. Wu H, Wang S, Fang $\mathrm{H}$, Zan $\mathrm{X}$, Zhang $\mathrm{J}$ and Wan $\mathrm{Y}$ : Chitosan-polycaprolactone copolymer microspheres for transforming growth factor- $\beta 1$ delivery. Colloids Surf B Biointerfaces 82: 602-608, 2011.

17. Zhao J, Fan X, Zhang Q, Sun F, Li X, Xiong C, Zhang C and Fan H: Chitosan-plasmid DNA nanoparticles encoding small hairpin RNA targeting MMP-3 and -13 to inhibit the expression of dedifferentiation related genes in expanded chondrocytes. J Biomed Mater Res A 102: 373-380, 2014.

18. Breyner NM, Hell RC, Carvalho LR, Machado CB, Peixoto Filho IN, Valério P, Pereira MM and Goes AM: Effect of a three-dimensional chitosan porous scaffold on the differentiation of mesenchymal stem cells into chondrocytes. Cells Tissues Organs 191: 119-128, 2010.

19. Muzzarelli RA, Greco F, Busilacchi A, Sollazzo V and Gigante A: Chitosan, hyaluronan and chondroitin sulfate in tissue engineering for cartilage regeneration: A review. Carbohydr Polym 89: 723-739, 2012 .

20. Zhou PH, Ma BL, Shi L, Xie T and Qiu B: Inhibition of interleukin-1 $\beta$-stimulated matrix metalloproteinases via the controlled release of interleukin-1Ra from chitosan microspheres in chondrocytes. Mol Med Rep 11: 555-560, 2015.

21. Andric SA, Janjic MM, Stojkov NJ and Kostic TS: Testosterone-induced modulation of nitric oxide-cGMP signaling pathway and androgenesis in the rat Leydig cells. Biol Reprod 83: 434-442, 2010.

22. Huang Y, Wu D and Fan W: Protection of ginsenoside Rg1 on chondrocyte from IL-1b-induced mitochondria-activated apoptosis through PI3K/Akt signaling. Mol Cell Biochem 392: 249-257, 2014.

23. Rasheed Z, Al-Shobaili HA, Rasheed N, Mahmood A and Khan MI: MicroRNA-26a-5p regulates the expression of inducible nitric oxide synthase via activation of NF- $\mathrm{KB}$ pathway in human osteoarthritis chondrocytes. Arch Biochem Biophys 594: 61-67, 2016

24. Yu SM and Kim SJ: Protein phosphorylation on tyrosine restores expression and glycosylation of cyclooxygenase-2 by 2 -deoxy-D-glucose-caused endoplasmic reticulum stress in rabbit articular chondrocyte. BMB Rep 45: 317-322, 2012.

25. Hwang HS and Kim HA: Chondrocyte apoptosis in the pathogenesis of osteoarthritis. Int J Mol Sci 16: 26035-26054, 2015.

26. Youle RJ and Strasser A: The BCL-2 protein family: Opposing activities that mediate cell death. Nat Rev Mol Cell Biol 9: 47-59, 2008.

27. Borner C: The Bcl-2 protein family: Sensors and checkpoints for life-or-death decisions. Mol Immunol 39: 615-647, 2003.

28. Loeser RF, Erickson EA and Long DL: Mitogen-activated protein kinases as therapeutic targets in osteoarthritis. Curr Opin Rheumatol 20: 581-586, 2008.

29. Chang JK, Chang LH, Hung SH, Wu SC, Lee HY, Lin YS, Chen $\mathrm{CH}, \mathrm{Fu}$ YC, Wang GJ and Ho ML: Parathyroid hormone 1-34 inhibits terminal differentiation of human articular chondrocytes and osteoarthritis progression in rats. Arthritis Rheum 60: 3049-3060, 2009.

30. Csaki C, Mobasheri A and Shakibaei M: Synergistic chondroprotective effects of curcumin and resveratrol in human articular chondrocytes: Inhibition of IL-1beta-induced NF-kappaB-mediated inflammation and apoptosis. Arthritis Res Ther 11: R165, 2009. 